\title{
MAGNETIC SHIELDING OF AN ELECTRON BEAMLINE IN A HADRON ACCELERATOR ENCLOSURE*
}

\author{
T.K. Kroc ${ }^{\#}$, C.W. Schmidt, A. Shemyakin \\ FNAL, Batavia, IL 60510, USA
}

\begin{abstract}
The Fermilab Electron Cooling Project requires the operation of a $4.34 \mathrm{MeV}$ electron beam in the same enclosure that houses the $120,150 \mathrm{GeV}$ Main Injector. Effective shielding of the magnetic fields from the ramped electrical bus ses and local static fields is necessary to maintain the high beam quality and recirculation efficiency required by the electron cooling system. This paper discusses the operational tolerances and the design of the beamline shielding, bus design, and bus shielding as well as experimental results from the prototype and final installation.
\end{abstract}

\section{INTRODUCTION}

The Electron Cooling Project is an upgrade to Fermilab's Recycler which shares an accelerator enclosure with the Main Injector. The Recycler ring serves as a storage and cooling ring for the antiproton production. Stacks of antiprotons are periodically transferred from the Accumulator a nd further cooled by a stochastic cooling system. Improvements in antiproton production continue to increase antiproton intensities and additional cooling will be required which will be provided by the E lectron Cooling system. Cooling the antiprotons requires that the electron and antiproton beams be brought into coincident trajectories with equal velocities. The cooling takes place in 10 two meter long solenoids. The magnetic field of the cooling solenoids guides the electrons. If the optics is properly matched at the entrance of the cooling solenoids, the electrons move in a laminar flow and are able to cool the antiprotons. The relative angular spread of the electrons needs to be less than 200 $\mu \mathrm{rad}$. Of this $50 \mu \mathrm{rad}$ is allocated to motion of the central beam trajectory through the cooling solenoid.

Figure 1 shows a cross section of the Main Injector tunnel which houses the Main Injector (MI), Recycler (RR), and the Electron Cooling (EC) beam lines. The R R and the EC cooling section run approximately 1 foot below the ceiling of the tunnel. The EC return line runs approximately 15 " above the MI line and passes just above a MI quadrupole which is outlined in gray. The bus for the dipoles of the Main Injector passes through this area and was carried in two condu ctors of opposite polarity with a current of up to 10000 amps each.

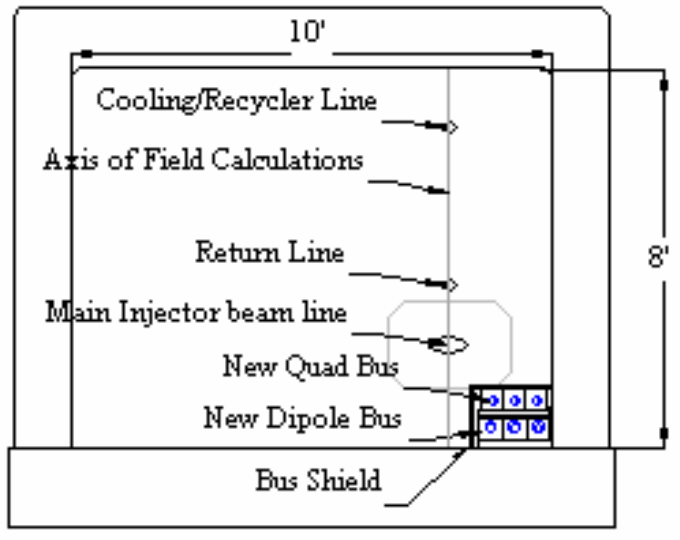

Figure 1 Physical layout of Main Injector enclosure housing Main Injector beam line, Recycler, and Electron Cooling cooling and return lines. Also shown is loc ation of electrical busses.

Two quad busses are also present with currents of 6000 7000 amps. All the busses have been relocated as shown in the lower right corner of the figure. The dipole bus was formerly located near where the new quad busses are. The quad busses were formerly where the return line is now located. (The entire Electron Cooling layout can be found in reference [1]). Figure 2 shows the magnetic fields measured two years ago at the height of the return line. Fields ranged from .5 to 1 Gauss.

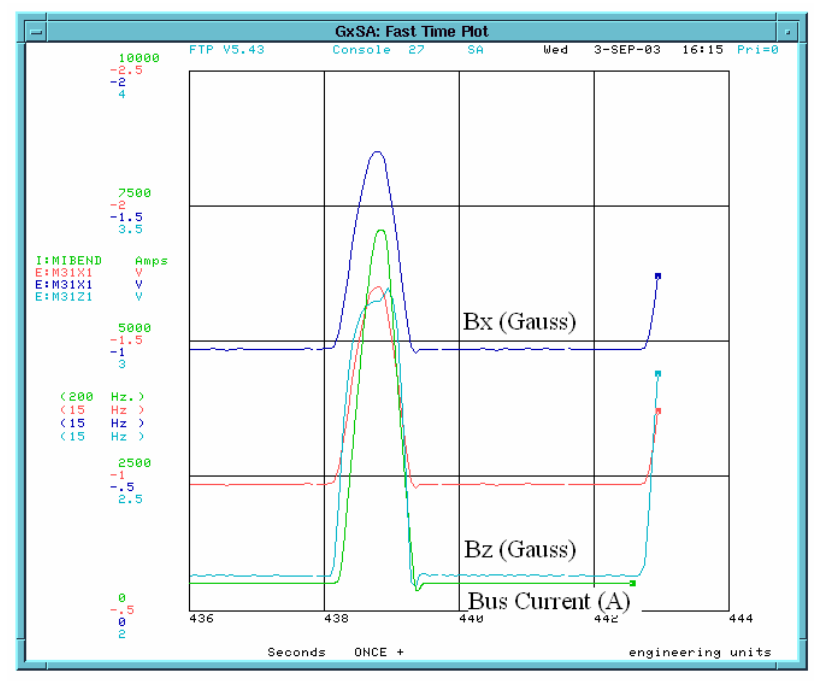

Figure $2 \mathrm{MI}$ ramp and measured magnetic fields at position of electron return line. Calibration for field measurements is $1 \mathrm{~V}=1$ Gauss.
*Operated by Universities Research Association Inc. under
76CH03000 with the United States Department of Energy.

${ }^{*}$ kroc@fnal.gov 
Electron Cooling uses a high current (.5A) electron beam to cool the antiprotons. The electron beam is generated by an electr ostatic accelerator (a Pelletron manufactured by NEC). To avoid damaging the beam line and prevent discharges of the Pelletron, the charge of the beam must be recirculated with relative losses of $3 \times 10^{-5}$ or less. Induced beam motion can reduce this efficien cy below the threshold for recirculation.

Experience with the Recycler showed [2] that initial attempts to shield the anti proton beam from the magnetic fields of the MI were not adequate and additional shielding had to be installed. Because they are $2000 \mathrm{t}$ imes lighter, electron beams require even more shielding.

\section{SHIELDING}

\section{Cooling section}

The cooling section provides the longitudinal magnetic field to guide the electrons. These solenoids are surrounded by three layers of concentric mu -metal shielding[3]. The design specified an initial mu of 11000 to provide a total magnetic attenuation for DC magnetic fields of 3000. This was verified by in -situ measurements using an external coil and a measurement system used for determining the quality of the solenoidal field[3]. These measurements found the shielding to be between 3000 and 5000.

\section{Transport lines}

The non -cooling beam li nes also need shielding. The lines not in the MI tunnel must be protected from the earth's magnetic field. Lines to and from the tunnel pa ss through a 7' steel shield wall. All non -cooling lines are shielded by a single layer of mu -metal constructed from an $80 \%$ nickel -iron-molybdemun alloy, .04" thick, with an initial magnetic permeability of greater than 25,000.

Particular attention was paid to the return line in the MI tunnel. While beam quality tolerances are not as stringent beyond the cooling section, the beam must be returned to the Pelletron with high enough efficiency to maintain recirculation and prevent damage to components.

Three sections of the return line are approximately 18 ' long. Each of these has 4 cylindrical shields constructed so that each quarter can telescope into another quarter. This is referred to as a "trombone" shield. Shorter sections of beamline are shielded by "cl amshell" shielding. These are two half cylinders that fit together with a captured edge.

The shields were tested with a spare MI quadrupole magnet at Fermilab's Magnet Test Facility. The two types of shields were each placed above the magnet so their centerline was approximately 15 " above the magnet center. Here the maximum external field of the quadrupole is about 10 Gauss. The trombone shielding reduced the field by a factor of $400-500$. The clamshell shields were tested in two orientations, with the sea m vertical and horizontal. With the seam oriented vertically the field was reduced by a factor of approximately 300 . When the seam was oriented horizontally the reduction factor was about 150 .

\section{FIELD REDUCTION}

\section{Bus geometry}

The installation of the electro $\mathrm{n}$ cooling line necessitated moving the quad busses. In addition it was decided to move the dipole busses also. These were moved as near to the floor as possible in order to maximize the distance between them and the electron beam lines.

The configuration of the busses was also changed. The magnetic field produced by 2 parallel conductors of opposite polarity decreases as $1 / \mathrm{r} \quad 2$. If one of the conductors is divided in half and is arraigned analogous to a linear electric quadrupole, the field decreases as $1 / \mathrm{r}{ }^{3}$. The effect of this is shown in figure 3 in curves $a$ and $b$. Additionally, the busses were placed behind steel shielding.

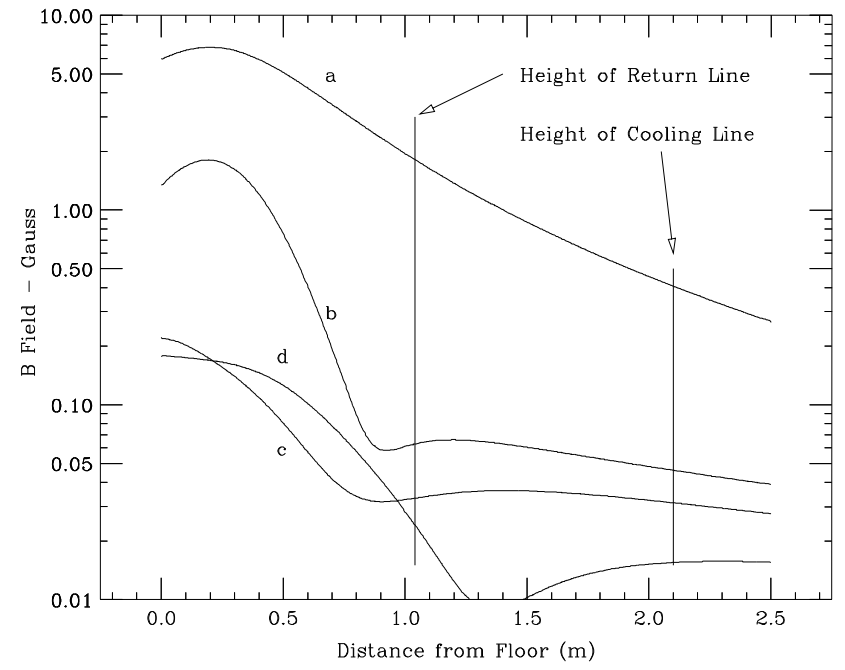

Figure 3. Magnetic fields along a vertical line in the plane of the beam lines in the MI enclosure due to the dipole and quad busses. Curve a is due to the original dipole bus. Curve $b$ is due to the dipole and quadrupole busses in a linear quadru pole configuration. Curves $\mathrm{c}$ and $\mathrm{d}$ are with two and three sided shielding around the busses respectively.

\section{Simulations}

The layout of these compon ents $\mathrm{w}$ as simulated in Opera[4] to estimate the magnetic fields that the electron beam would encounter. The fields were calculated along a vertical line passing through the MI, RR, and EC cooling and return lines, noted in figure 1 by the grey line. The results of this calculation are shown in figure 3 . Curve a is the field from the original 2 conductor dipole bus in its original location. This provides a benchmark for the other configurations. Curve $b$ is the field for the dipole and quadrupole busses in th eir linear quadrupole configurations. The orientation of the quadrupole polarities relative to the dipole polarities has been chosen so that the two cancel each other as much as possible. Curve $\mathrm{c}$ shows the effect of placing quarter inch thick steel plate o $\mathrm{n}$ two sides as shown in figure 1 to provide shielding for the magnetic fields. Curve $d$ is a three sided shield which includes a pl ate on the floor. While $\mathrm{d}$ is 
superior we chose to use the two sided shield for ease -ofinstallation and time constraints.

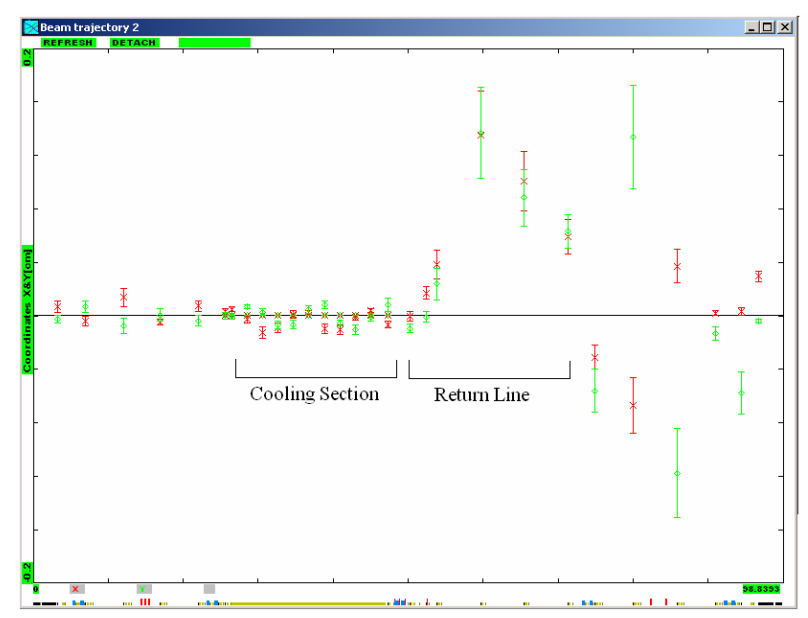

Figure 4 Example of trajectory deflection due to MI ramp. Full scale on y-axis is $\pm .2 \mathrm{~cm}$.

\section{OPERATIONAL EXPERIENCE}

After a couple years of development as a prot otype in a different enclosure (without transient magnetic fields) the EC system has been installed in the MI tunnel and commissioning has begun. Our initial experience indicates that the shielding up to, and through the cooling section is adequate. Figure 4 shows the induced deflection due to the MI ramp. The plot shows the difference in beam positions when measured at a time with no induced fields and a time when the MI ramp is approximately $25 \%$ its peak value. (The $x$ axis of the plot is the longitudinal coordinate of the beam line from the Pelletron on the left through the line and back to the Pelletron on the right . The $\mathrm{y}$ axis is the displacement, $\pm .2 \mathrm{~cm}$ full scale, for $\mathrm{x}-$ red and y - green.) The variations through the cooli ng section are on the order of $50 \mu \mathrm{rad}$.

The return line shows significant changes in beam trajectory. Because of the difficulty in designing shields for complex vacuum elements, there are a few areas in the return line that have sizable gaps in the shielding. In particular, an are a of approximately 1 meter in length , including a multiwire beam profile monitor, is unshielded. It is near the beginning of the return line. We have been able to observe beam motion of a few millimeters in the return line due to the MI ramp (figure 5). These data were taken by varying the trigger for the data collection. As the trigger was moved along the time line of the ramp, the measurements sampled the beam position at various values of bus current and correspondingly the magnetic field. Because the cu rrents are sampled on both the rising and falling edges of the ramp, a hysteresis loop is formed. Most of the motion is induced near location of the multiwire. Work is underway to correct this.

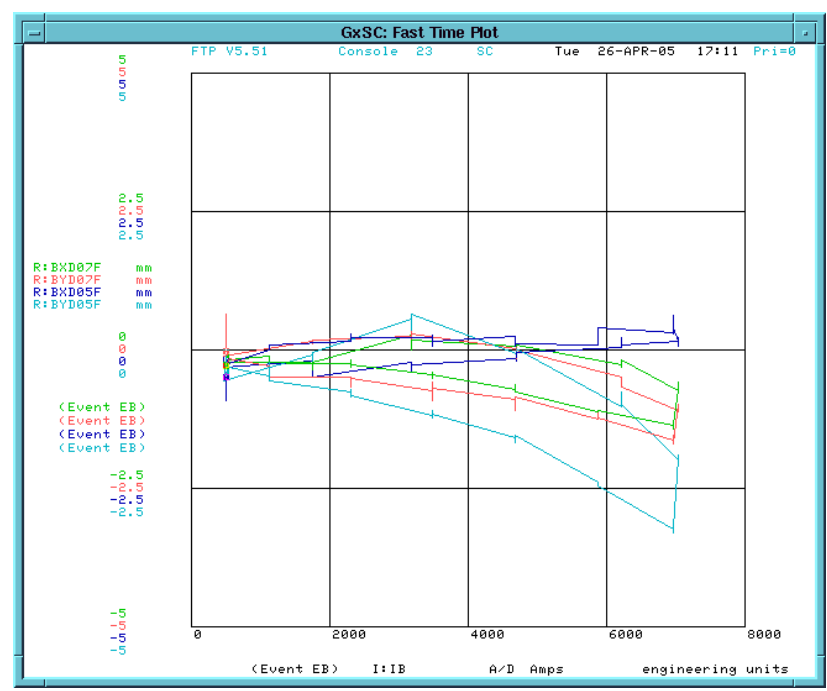

Figure 5 Beam motion due to induced magnetic fields from the MI measured near the end of the EC beam line. Shown are $\mathrm{x}$ and $\mathrm{y}$ positions at two locations prior to re entering the Pelletron. The $\mathrm{x}$-axis is the current in the dipole bus as the trigger event is varied along the timeline of the ramp. The lower portion of each loop is the rising edge of the ramp and the upper portion is the falling edge of the ramp.

\section{CONCLUSIONS}

A large portion of the Fermilab Electron Cooling beam line is located in the same enclosure as the Main Injector accelerator. The induced magnetic fields from the MI dipole and quadrupole busses are a significant issue for the electron beam. Magnetic shielding of the electron beam line is very important and has been accomplished in most areas. Most critical is the cooling solenoid which has been effe ctively shielded by a three layer system of mu metal. Beam motion in the cooling section meets our tolerances. The rest of the line uses a single layer of mu metal. The induced magnetic fields have been reduced as much as possible through the configuration of the electric busses in the enclosure. Magnetically induced beam motion has been observed after the cooling section and is attributed to a few areas where physical geometry made it difficult to shield. Since this motion may limit our recirculation efficiency, further attention is being given to these areas.

\section{REFERENCES}

[1] S. Nagaitsev, et. al. "Commissioning of Fermilab's Electron Cooling System for 8-GeV Antiprotons," these proceedings.

[2] S. Nagaitsev, et.al., "Experience with Magnetic Shielding of a Large Scale Accelerator," PAC'01, Chicago, June 2001, p. 3371.

[3] AC. Crawford, et. al.,"Field Measurements in the Fermilab Electron Cooling Solenoid Prototype," FERMILAB-TM-2224, Sept., 2003.

[4] OPERA 1.5, Vector Fields Limited, Oxford, England, 1995. 